Campos Neutrais - Revista Latino-Americana de Relações Internacionais Vol. 1, No 2, p. 8-25, Maio - Agosto de 2019

\title{
Preferencias socioeconómicas por costos ambientales en la Región Oeste del Lago de Cuitzeo, Michoacán, México
}

\author{
Rafael Trueba Regalado ${ }^{1}$ \\ Carlos Francisco Ortiz Paniagua ${ }^{2}$
}

Resumem: El presente artículo tiene por objetivo desarrollar un análisis descriptivo de las preferencias sociales para valorar económicamente la salud poblacional por el arribo de tolvaneras en la Región Oeste del Lago de Cuitzeo (ROLC), Michoacán, México. Esta investigación parte desde el enfoque teórico de la economía ambiental para aplicar el método de valoración contingente (MVC), con la finalidad de cuantificar el costo defensivo total al que incurre la población para evitar una externalidad negativa, derivada del deterioro ambiental que presenta el lacustre. Aunado a ello, también se busca determinar una posible disposición de la población a aceptar una compensación (DAC) por la disminución de su bienestar y una disposición a pagar (DAP) para que se realicen acciones que mejoren las condiciones ambientales del cuerpo de agua. Los resultados indican que el costo defensivo en salud es elevado en referencia al ingreso de la población entrevistada. También se identificó que existe una disposición a ser compensado por la mayoría de las personas consultadas, así como una disposición a pagar. Esta información pretende proporcionar elementos de gestión para establecer mecanismos compensatorios y de restauración para reducir costos futuros.

Palabras clave: externalidad, costos defensivos, valoración contingente, disposición a aceptar, disposición a pagar.

\section{Socioeconomics preferences by environmental costs in the West Region of Cuitzeo's Lake, Michoacan, Mexico}

\begin{abstract}
This article aims to develop a descriptive analysis of social preferences to economically value population health by the arrival of dust storms in the Western Region of Cuitzeo's Lake (WRCL), Michoacan, Mexico. This research stars from the theoretical approach of environmental economics to apply the contingent valuation method (CVM) with the purpose of quantifying the total defensive cost incurred by the population to avoid a

1 Doctor en Ciencias del Desarrollo Regional por el Instituto de Investigaciones Económicas y Empresariales de la Universidad Michoacana de San Nicolás de Hidalgo. Profesor Catedrático en el Instituto Tecnológico y de Estudios Superiores de Monterrey, Campus Morelia. Correo electrónico: trueba_59@hotmail.com

2 Doctor en Ciencias del Desarrollo Regional. Profesor-Investigador de tiempo completo en el Instituto de Investigaciones Económicas y Empresariales de la Universidad Michoacana de San Nicolás de Hidalgo. Correo electrónico: carlinortizpaniagua@gmail.com
\end{abstract}


negative externality derived from the environmental deterioration that the lake presents. In addition, it also seeks to determine a possible willingness of the population to accept a compensation (WTA) for the reduction of their welfare and a willingness to pay (WTP) for actions to improve the environmental conditions of the water body. The results indicate that the defensive cost in health is high in reference to the income of the population interviewed. It was also identified that there is a willingness to be compensated by most of the people consulted, as well as a willingness to pay. This information aims to provide management elements to establish compensatory and restoration mechanisms to reduce future costs.

Keywords: Externality, defensive costs, contingent valuation, willingness to accept, willingness to pay.

\section{Introducción}

La economía ambiental trata el estudio de los problemas ambientales con la perspectiva e ideas analíticas de la ciencia economía. Estudia cómo y por qué "las personas", empresas, organizaciones o agencias gubernamentales, etc. toman decisiones sobre el uso de recursos naturales valiosos" (FIELD, 1996:1). Un ejemplo de éstos se encuentra en aquellos que son indispensables en la vida cotidiana de una persona, que normalmente se ven como recursos de uso común pero poco valorados en la práctica como el agua de un lago y las externalidades negativas que puede causar el deterioro de éste en la salud de poblaciones ribereñas, como sucede en la ROLC.

El lago de Cuitzeo presenta un proceso de deterioro ambiental y desecación en su vaso Oeste, atribuido principalmente a las actividades productivas humanas que se realizan en la cuenca que lo integran y las descargas residuales provenientes de la ciudad de Morelia (Bravo-Espinosa et al., 2008). Durante los primeros meses del año, esta parte del lacustre alcanza su proceso de estiaje más bajo y todos los contaminantes que tiene el agua se asientan en el suelo (FERNÁNDEZ, SOMMER, CRAM, PONCE DE LEÓN y BECERRA, 2010).

La velocidad y dirección del viento favorecen la aparición de uno de los efectos más dañinos, esto es, la generación de tolvaneras que cubren a las poblaciones ribereñas, principalmente en Capacho (Huandacareo), Doctor Miguel Silva y Jéruco (Cuitzeo), con el consiguiente incremento de las enfermedades ${ }^{3}$ respiratorias, gastrointestinales, y dermatológicas (CHACÓN, ROSAS y ALVARADO, 2007).

\footnotetext{
3 Existen registros oficiales que indican que en la ROLC durante el periodo 2005-2015 las principales enfermedades que se han detectado en la población en relación con el proceso de las tolvaneras son: las infecciones respiratorias aguas y las infecciones intestinales por organismos. Las primeras presentan 97,026 casos en total por los cuatro municipios que se están abordando y las segundas registran la cantidad de 11,288 habitantes (Secretaria de Salud en Michoacán, 2015).
} 
Campos Neutrais - Revista Latino-Americana de Relações Internacionais Vol. 1, No 2, p. 8-25, Maio - Agosto de 2019

Por ello, este artículo busca contribuir con un análisis descriptivo de frecuencias en cuanto a las preferencias sociales por costos ambientales para recibir una compensación económica ante la externalidad negativa que sufren los habitantes interrogados, y a su vez, la disposición que tienen para pagar por realizar acciones de recuperación ambiental para el lacustre. Esto busca construir mecanismos compensatorios y de restauración de restauración en la ROLC.

\section{La visión de la economía ambiental}

La presente investigación se apoya en los fundamentos teóricos de la economía ambiental. El surgimiento de este enfoque se sitúa habitualmente en la década de los años cincuenta, y, en concreto, en la formación de la organización Resources for the Future, en 1952 (FIELD, 1996). Esta rama de la economía "se ubica en el campo de la microeconomía. Se concentra principalmente en cómo y por qué las personas toman decisiones que tienen consecuencias ambientales. Además, estudia cómo se pueden cambiar las políticas e instituciones económicas con el propósito de equilibrar un poco más esos impactos ambientales, con los deseos humanos y las necesidades del ecosistema en sí mismo" (FIELD, 1996:1).

La economía ambiental ha abierto la oportunidad de poder valorizar los bienes ambientales para tratar de conocer cuánto vale cada uno de éstos (que normalmente se desconoce) y ante ello, se toma la decisión irracional de dañarlos causando desequilibrios en su entorno natural. Una pregunta relevante en cuanto a este tipo de estudios es la que plantea FIELD (1995:2): “'Por qué las personas se comportan de manera tal que ocasionan la destrucción del ambiente? Existen varias clases de respuestas para esta pregunta. Una puede ser la siguiente: la degradación ambiental surge a partir del comportamiento humano que carece de ética o moral'. Se puede pensar que el ser humano carecer de estos valores, sin embargo, la razón es que busca generar un beneficio personal a costa de perjudicar otros bienes naturales, porque le resulta más fácil actuar de esa manera irracional que pensar cómo podría actuar sin dañar nada.

Entonces, aquí surge la importancia teórica que posee la economía ambiental, basada en estudiar habitualmente dos cuestiones: el problema de las externalidades y la asignación intergeneracional óptima de los recursos agotables. PIGOU (1920) y COASE (1960) sientan las bases conceptuales para la discusión sobre cómo este enfoque puede ayudar a generar información que permita contribuir para resolver el problema las externalidades (AGUILERA y ALCÁNTARA, 1994). 
Campos Neutrais - Revista Latino-Americana de Relações Internacionais Vol. 1, No 2, p. 8-25, Maio - Agosto de 2019

Para AGUILERA y ALCÁNTARA (1994:11), “Coase señala que las conclusiones derivadas del tratamiento de Pigou conducen a los economistas a tres opciones posibles resultados. Éstos son: a) que la empresa que contamine responda por los perjuicios ocasionados, b) que la empresa pague un impuesto en función de los daños ocasionados y c) que se aparte a la fábrica de los distritos residenciales".

Teóricamente parecen ser muy acertados los planteamientos propuestos por Pigou, sin embargo, al momento de querer internalizar las externalidades que se derivan por la contaminación de una empresa, entra en tela de juicio el cómo es posible valorar el daño ocasionado a un recurso finito como el agua.

Gracias a numerosos estudios, en la actualidad se comienza a romper esa barrera para ir adoptando métodos que permiten conocer ese valor y con ello, poder disminuir las externalidades negativas, mediante una adecuada intervención por parte del Estado, a través de diferentes mecanismos. Esto representa un primer acercamiento para disminuir el impacto ambiental y los efectos negativos que se pueden desencadenar.

Centrando el análisis de manera puntual, Pigou plantea una manera de corregir las externalidades, indicando que esto se presenta cuando no existen relaciones contractuales entre el causante y los afectados por la externalidad. Entonces, el Estado, si así lo desea, puede impulsar o restringir de un modo extraordinario las inversiones en dichas actividades. Las formas más conocidas para impulsar y restringir las inversiones pueden revestir carácter de primas o impuestos (AGUILERA y ALCÁNTARA, 1994).

Estos mecanismos en la actualidad son los que permiten diseñar una política ambiental coherente por el daño al medio ambiente, ya que con base en esos postulados teóricos las externalidades se pueden internalizar y quedar obsoletas si la empresa o individuo que perjudican un bien ambiental pagan por su proceso de recuperación. De aquí surge la importancia de la economía ambiental en la actualidad, ya que es la única rama de la economía que permite llevar a cabo estudios reales y cuantificables en términos monetarios, aunque esto continúa siendo tema de discusión. Sin embargo, es el enfoque que se adoptó para el desarrollo de la investigación en la ROLC.

\section{Valoración económica de las externalidades en la Región Oeste del Lago de Cuitzeo}

Se entiende por externalidades ambientales a las interacciones que surgen entre consumidores y/o productores en el uso de los bienes que proporciona el medio ambiente. Se trata, por tanto, de un concepto útil para definir las relaciones entre productores y/o consumidores que no pasan por el mercado (LABANDEIRA et al., 2007). 
Campos Neutrais - Revista Latino-Americana de Relações Internacionais Vol. 1, No 2, p. 8-25, Maio - Agosto de 2019

Existen diversos tipos de externalidades, esto en relación a la naturaleza de los efectos de la externalidad, ésta puede ser positiva si la acción que no pasa por el mercado supone un aumento de bienestar; y negativa si supone una reducción mercado (LABANDEIRA et al., 2007). En el caso de la problemática que se está analizando la externalidad es negativa, ya que el deterioro del lago de Cuitzeo está generando una pérdida de bienestar para la población en cuestiones de salud. Las externalidades se consideran en diferentes tipos dependiendo de los agentes que estén involucrados, a continuación, se hace mención a éstas, resaltando la que está vinculada con la problemática del estudio:

a) La externalidad entre productores: se formula cuando la producción y los beneficios de una empresa dependen de las acciones tomadas por otros productores, siendo también conocidas como externalidades de producción al sufrirlas un productor (LABANDEIRA et al., 2007).

b) La externalidad entre consumidores: se desarrolla cuando la utilidad de un consumidor está en función de las decisiones adoptadas por otro consumidor, siendo también conocidas como externalidades de consumo al sufrirlas un productor (LABANDEIRA et al., 2007).

c) La externalidad entre consumidores y productores: en este caso, la utilidad de los individuos está en función del nivel de producción de alguna empresa (entendiendo esto como externalidad de consumo), o las posibilidades de producción dependen del consumo o acciones de algunos individuos (representa una externalidad de productor). Este tipo de externalidades pueden ser positivas o negativas para productores y consumidores, un ejemplo para los primeros es el beneficio que puede brindar el comportamiento de un consumidor para generar las posibilidades de producción en cuanto a materiales reciclados. Para las externalidades negativas en referencia a los consumidores, el ejemplo son los efectos negativos sobre la salud de los habitantes de un territorio por las emisiones contaminantes de una empresa (LABANDEIRA et al., 2007).

Las externalidades que se relacionan con la problemática de esta investigación, son entre consumidores y productores, ejemplificando lo mencionado en párrafos anteriores la relación se da en la siguiente forma: el agente productor de la externalidad negativa que se encuentra en lago de Cuitzeo (que ha propiciado la desecación de éste y con ello la aparición de tolvaneras) la realizan las empresas y los habitantes de la parte alta de la cuenca del lago, en particular derivan de Morelia, ciudad que concentra la mayor dinámica económica y 
Campos Neutrais - Revista Latino-Americana de Relações Internacionais Vol. 1, No 2, p. 8-25, Maio - Agosto de 2019

poblacional. El consumidor que está sufriendo las externalidades negativas son los habitantes de la ROLC, en específico en su salud. De aquí surge la importancia de entender como plantear la relación entre consumidores y productores en cuanto a las externalidades, ya que esto permite tener el panorama bien trazado para generar la valoración económica mediante los métodos que aporta la economía ambiental, en este caso bajo el MVC.

\section{Los fallos del mercado en la valoración económica del medio ambiente}

El mercado es una institución que tiene como su principal característica la asignación de los recursos económicos de forma descentralizada, sin la necesidad de que intervengan mecanismos de supervisión de los flujos de materiales y de dinero que participan en los procesos de intermediación (LABANDEIRA et al., 2007).

En ese sentido, FIELD (1996:79) hace hincapié en que: “el mercado es una institución en la cual vendedores y compradores (de bienes de consumo, factores de producción, etc.) realizan intercambios mutuamente basándose en acuerdos". En el mercado se presenta la posibilidad de intercambiar bienes y servicios por medio de flujos monetarios, que sirven de medio de cambio y facilitan las transacciones en la actualidad. Por ello, LABANDEIRA et al. (2007:68) señala que: "el funcionamiento del mercado se puede visualizar como un proceso en el que se realiza una subasta continua de precios hasta que la oferta y la demanda se equilibran y el mercado se vacía".

Con ello, se asume teóricamente que el mercado es una institución que resulta útil para aumentar el bienestar colectivo en una sociedad, que parece ser perfecta. Sin embargo, el mercado no es una institución perfecta, porque los supuestos económicos no generan una asignación de recursos económicos en todos los escenarios de una sociedad. El mercado puede fallar cuando no existe una asignación eficiente de los recursos. A estos incumplimientos se les conoce como fallos de mercado (LABANDEIRA et al., 2007).

El mercado falla porque en la realidad no cumple con los supuestos en relación al teorema de la economía del bienestar, según LABANDEIRA et al. (2007) éstos son:

a) El mercado es perfecto y cuenta con una adecuada definición de los derechos de propiedad. En donde los agentes que lo integran pueden realizar libremente intercambios para toda transacción.

b) Los consumidores y productores tienen un comportamiento competitivo, realizando planes de optimización racionales, los cuales plantean máximos beneficios y mínimos costos.

c) Los precios son conocidos por todos los agentes económicos, tanto empresas como 
Campos Neutrais - Revista Latino-Americana de Relações Internacionais Vol. 1, No 2, p. 8-25, Maio - Agosto de 2019

consumidores. La información es perfecta.

d) Los costos de transacción son escasos y la fijación de precios no supone el empleo de recursos.

Al momento de querer aplicar todos estos supuestos en la realidad se observa que el mercado es vulnerable ante todos estos escenarios. En el análisis económico para valorar el medio ambiente posee un problema y es que la mayoría de los bienes que lo integran no se encuentran dentro de un mercado. En esa orientación, se concibe que los problemas ambientales se vinculan a las fallas que éste presenta. Carencia de información (esta no es completa o perfecta), diseños institucionales (no hay derechos de propiedad) y de política que se traducen en costos de los agentes que los ocasionan hacia otros sectores (LABANDEIRA et al., 2007; PEARCE, 1976).

La información que pueden tener los agentes sobre los problemas ambientales no es perfecta, ya que normalmente las personas desconocen los efectos ambientales que generan las decisiones privadas de consumo y/o de producción. Para los bienes ambientales, como el aire, el agua, bosques etc. es complicado determinar quién es el propietario de estos recursos o quién posee los derechos de propiedad. Por esto, se argumenta que para la mayoría de los bienes ambientales los mercados no existen o no están bien definidos (LABANDEIRA et al., 2007).La problemática bajo estudio es un ejemplo del desconocimiento existente que tiene la sociedad por el valor de los bienes ambientales, dejando de considerar las consecuencias que la contaminación del lago de Cuitzeo generada por otros agentes y que gracias a las decisiones de éstos se ha desencadenado un problema de salud pública.

\section{Materiales y métodos}

En la figura 1 se observa la localización geográfica del LC en el noroeste de Michoacán, México. Éste se ubica en las coordenadas extremas sobre el sistema de proyección cartográfica Universal Transversal de Mercator (UTM) 0256900 E; 2203132 N y 0307561 E y 2202055 N (CHACÓN, ROSAS y ALVARADO, 2007). 
Campos Neutrais - Revista Latino-Americana de Relações Internacionais Vol. 1, No 2, p. 8-25, Maio - Agosto de 2019

FIGURA 1 - Localización geográfica de la zona de estudio en el estado de Michoacán, México

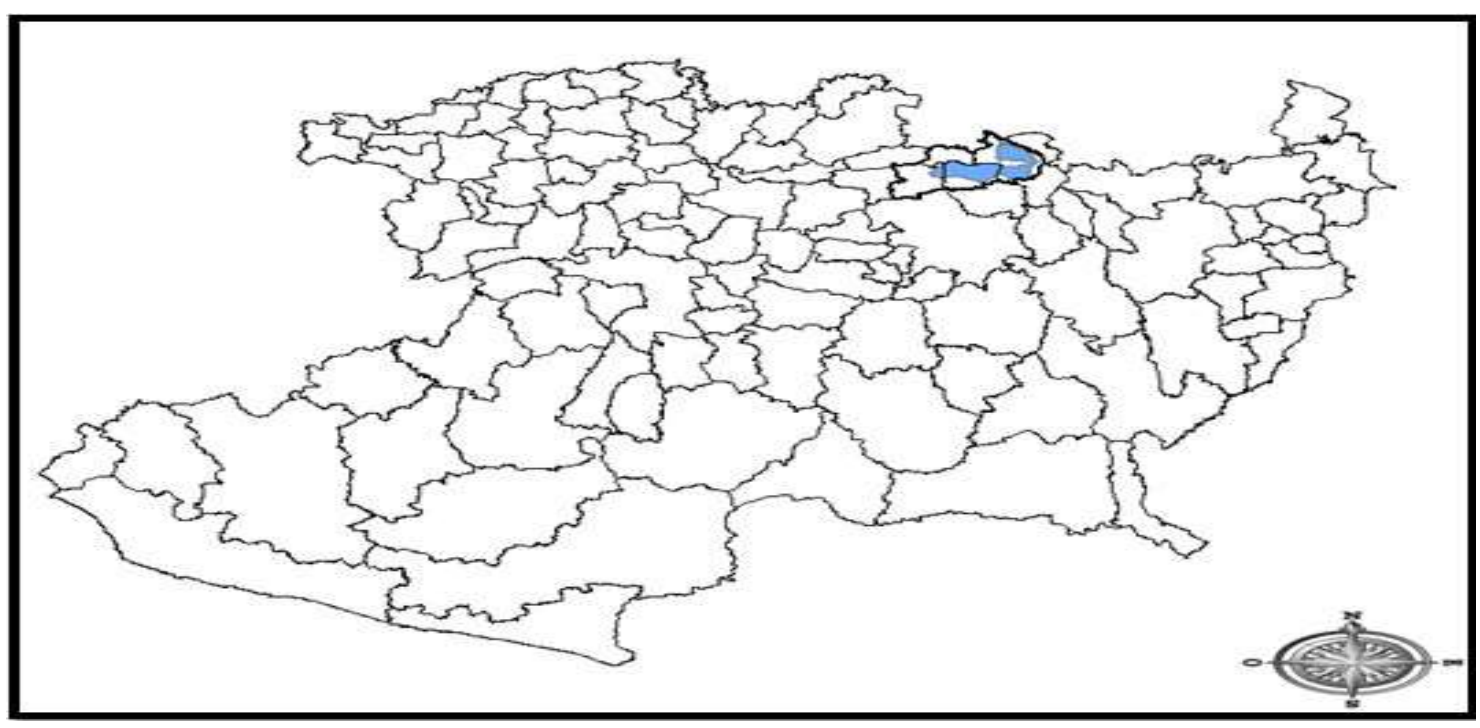

FUENTE: Extraído de: TRUEBA y ORTIZ, 2019.

La región de estudio (ROLC) está compuesta por los municipios ribereños perjudicados directamente por las externalidades negativas en relación al deterioro ambiental del lacustre, estos son: Copándaro, Cuitzeo, Chucándiro y Huandacareo. La zona de estudio está ubicada en el noroeste del estado de Michoacán (ver figura 2). La extensión territorial de la región es de $685.96 \mathrm{Km}^{2}$, esto representa para el estado de Michoacán únicamente el $1.17 \%$ de la superficie, siendo el municipio de Cuitzeo el que aporta mayor número de kilómetros registrando de cifra de $225.17 \mathrm{Km}^{2}$.

FIGURA 2 - Distribución espacial de las localidades urbanas y rurales en la región Oeste del Lago de Cuitzeo

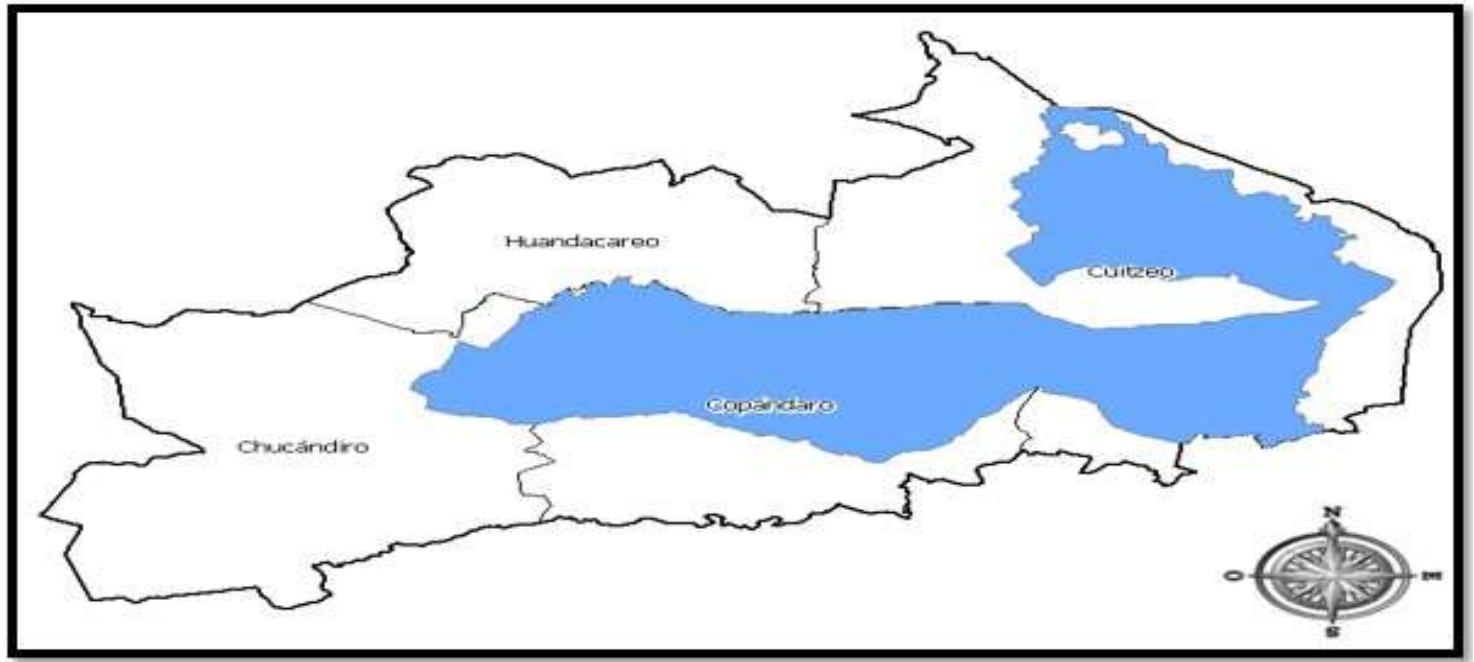

FUENTE: Elaboración propia con base en INEGI, 2010. 
El foque metodológico de esta investigación se fundamenta en la aplicación del $\mathrm{MVC}^{4}$. Éste se distingue por ser un método directo o hipotético de la economía ambiental para generar un estudio como el que se desarrolló. En otras palabras, de los métodos indirectos u observables éste tiene como objetivo que las personas declaren sus preferencias con relación a un determinado bien o servicio ambiental, en lugar de realizar estimaciones sobre conductas que se observan en el mercado.

En esa dirección, se elaboró y se aplicó en campo un cuestionario piloto previo a la realización del instrumento definitivo para detectar las fallas que éste podría presentar o la ausencia de compresión en cuanto a los cuestionamientos y la forma de capturar las respuestas. Todo esto con la finalidad de simular mercados hipotéticos.

Una vez que se obtuvo el cuestionario definitivo para el levantamiento de la información en campo, se aplicó a 272 personas (este dato fue calculado para una muestra representativa con $95 \%$ de confianza ${ }^{5}$ ) que habitaban en la zona de estudio para calcular los datos que se buscaban construir. La unidad de análisis fue la vivienda y la información se recolectó en las localidades más afectadas por las tolvaneras; Jéruco (66 cuestionarios), Doctor Miguel Silva (66 cuestionarios) y Capacho (140 cuestionarios). Es conveniente mencionar que se entrevistó a 215 hombres y 57 mujeres.

El instrumento de la investigación se dividió en 6 secciones (conocimiento del problema, costos defensivos en salud, disposición a ser compensado por los problemas en salud derivados de las tolvaneras, disposición a contribuir para realizar mejoras ambientales, compromiso ecológico por el mejoramiento ambiental del lacustre y las condiciones socioeconómicas del entrevistado). El cuestionario contenía 41 preguntas de las cuales 22 son cerradas y 19 abiertas. Sin embargo, en este documento no se analiza el compromiso ecológico.

\section{Análisis de los resultados}

Costos defensivos en salud de los entrevistados pertenecientes a la Región Oeste del

\section{Lago de Cuitzeo}

Después de capturar la información que se recolectó en campo aplicando el

\footnotetext{
4 Los antecedentes teóricos del MVC están ampliamente descritos en la literatura del tema, por ello, no serán explicados en este artículo. Sin embargo, se recomienda al lector los trabajos de RIERA (1994) o AZQUETA (2002). Aunado a ello, si se pretende conocer cómo se ha utilizado el método para valorar bienes ambientales, se sugiere consultar los trabajos de MARTÍNEZ-PAZ, ALMANSA-SÁEZ y PERNI-LLORENTE (2010) y (2011).

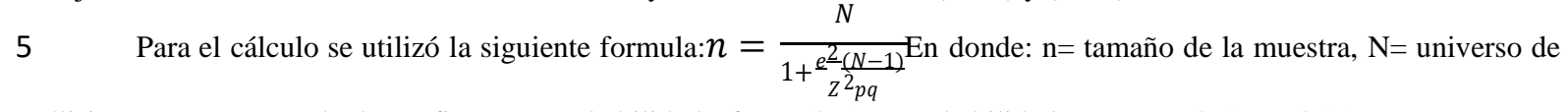
análisis, $\mathrm{e}=$ error, $\mathrm{z}=$ grado de confianza $\mathrm{p}=$ probabilidad a favor $(0.5) \mathrm{q}=$ probabilidad en contra $(0.5) \mathrm{pq}=0.25$
} 
Campos Neutrais - Revista Latino-Americana de Relações Internacionais Vol. 1, No 2, p. 8-25, Maio - Agosto de 2019

cuestionario, se realizó un análisis descriptivo mediante frecuencias, obteniendo los siguientes resultados preliminares. De 272 personas que fueron entrevistadas el 88\% considera que el cambio climático es muy importante. En cuanto a la mala gestión/escasez del agua 87\% de los habitantes creen que es muy importante. El siguiente tema fue la degradación del entorno natural, 95\% de las personas opinaron que es muy importante, resaltando en particular la contaminación y disminución de recurso hídrico en el lacustre. En esa dirección, la producción y mala gestión de residuos, resulta ser muy importante para los habitantes registrando $83 \%$.

La preocupación de los entrevistados en cuanto al proceso de contaminación del lago y la probabilidad de que desaparezca en un futuro, convirtiéndose en un foco de infecciones para ellos es notoria, por la incertidumbre que tienen respecto al tema, ya que $91 \%$ eligieron la respuesta mucho. En la charla se comentó que esto ya está pasando en la actualidad, pero no es algo constante que suceda cada año y el miedo existente es porque se puede convertir en algo permanente ese comportamiento de desecación total. Estos comentarios, se vinculan con el grado de conocimiento que tiene la población en cuanto a la problemática del estudio, en donde $50 \%$ conocen algo sobre el tema y $45 \%$ de los habitantes entrevistados conocen bastante sobre el tema.

Es muy importante la vinculación del gobierno federal para resolver la problemática, así lo consideraron el $85 \%$ de los entrevistados, comentaron que la participación de este agente es necesaria para que cualquier estrategia diseñada a favor del lago pueda contar con recursos etiquetados. El gobierno estatal y municipal, también se consideran muy importantes, registrando el primero $84 \%$ y el segundo $80 \%$.

Los entrevistados mencionaban que se necesita de las autoridades federales en mayor medida, porque los niveles de gobierno estatal y municipal no han planteado alguna propuesta de solución real al problema, dejando que la situación continué; incluso se comentaba que existe corrupción en las decisiones de los encargados, ocasionando una nula realización de actividades para mejorar las condiciones del lago, porque el dinero etiquetado para esto se lo han robado o se ha destinado para otros rubros. Por ello, hay pérdida de confianza y poca credibilidad en las instituciones estatales y municipales.

Por otro lado, la participación ciudadana también debe aplicarse, ya que se argumentaba que ellos tienen la intención de ayudar para realizar acciones a favor de la recuperación del lago para disminuir de las tolvaneras, pero no saben cómo ejecutarlas para tener éxito y requieren de expertos que puedan orientarlos en el tema

En la tabla 1 se contempla la cantidad promedio de personas que se enferman con 
Campos Neutrais - Revista Latino-Americana de Relações Internacionais Vol. 1, No 2, p. 8-25, Maio - Agosto de 2019

mayor frecuencia en un hogar cuando se presentan las tolvaneras, este comportamiento se ubica en un rango de $2(27 \%)$ a $3(20 \%)$. Resaltando que hay hogares que registran $4(10 \%)$ o $5(7 \%)$ habitantes como los siguientes valores más frecuentas hasta contemplar 12 habitantes enfermos. Por otro lado, $19 \%$ de los entrevistados no han registrado estos síntomas en su salud. En ocasiones, se mencionaba que esto sucede así porque preferían irse del lugar en cuanto arriba el polvo o preferían no salir de sus casas durante el día.

TABLA 1 - Personas enfermas cuatrimestre/hogar

\begin{tabular}{|c|c|c|c|c|}
\hline & Frecuencia & Porcentaje & Porcentaje válido & Porcentaje acumulado \\
\hline & 35 & 12.09 & 15.09 & 15.09 \\
\hline 2 & 72 & 26.05 & 32.07 & 48.06 \\
\hline 3 & 53 & 19.05 & 24.01 & 72.07 \\
\hline 4 & 28 & 10.03 & 12.07 & 85.05 \\
\hline 5 & 19 & 7.00 & 8.06 & 94.01 \\
\hline 6 & 8 & 2.09 & 3.06 & 97.07 \\
\hline 7 & 2 & .07 & .09 & 98.06 \\
\hline 8 & 2 & .07 & .09 & 99.05 \\
\hline 12 & 1 & .04 & .05 & 100.00 \\
\hline Total & 220 & 80.09 & 100.00 & \\
\hline No enfermos & 52 & 19.01 & & \\
\hline Total & 272 & 100.00 & & \\
\hline
\end{tabular}

FUENTE: Elaboración propia con base al trabajo de campo.

En la tabla 2 se muestran las enfermedades que tiene mayor incidencia, éstas son las respiratorias y oftalmológicas. Las primeras oscilan entre $1(28 \%)$ y 2 (30\%) casos por vivienda. Las segundas también registran $1(14 \%)$ y $2(9 \%)$ observaciones. Además de esto, la población también está resultando perjudicada por cuestiones

Además de esto, la población también está resultando perjudicada por cuestiones estomacales, alergias en la piel y dolores de cabeza ${ }^{6}$.

TABLA 2 - Personas enfermas cuatrimestre/hogar

\begin{tabular}{|l|c|c|c|c|c|}
\hline Variable (enfermedades) & Media & Mediana & Moda & Mínimo & Máximo \\
\hline Respiratorias & 2.04 & 2 & 2 & 0 & 6 \\
\hline Estomacales & 1.56 & 1 & 1 & 0 & 4 \\
\hline Oftalmológicas & 1.74 & 2 & 1 & 0 & 5 \\
\hline
\end{tabular}

FUENTE: Elaboración propia con base al trabajo de campo.

Ellos argumentaban que estos síntomas están vinculados al polvo porque únicamente los resienten sobre los primeros 4 meses del año, periodo en donde se presenta el arribo de éste a las localidades. Otro aspecto mencionado fue que los familiares provenientes de

6 Esta información brinda elementos para ir identificando que existe un problema de salud pública en los municipios ribereños al lago, por ello, se debe exhortar a las autoridades encargadas del tema para hacer frente ante la contingencia ambiental que se presenta: Secretaría de Salud Pública de Michoacán, Consejo Nacional del Agua (CONAGUA) y la Secretaria de Medio Ambiente, Cambio Climático y Desarrollo Territorial (SEMACCDET). 
Campos Neutrais - Revista Latino-Americana de Relações Internacionais Vol. 1, No 2, p. 8-25, Maio - Agosto de 2019

Estados Unidos se enferman con mayor facilidad cuanto están de visita porque no están acostumbrados al problema.

Por lo anterior, en la figura 4 se indica que $75 \%$ de los entrevistados han tenido la necesidad de asistir a consulta médica por las enfermedades mencionadas, con una frecuencia de $1(18 \%)$ a $2(29 \%)$ ocasiones y en segundo término acuden en un rango de $3(13 \%)$ a $5(3 \%)$ resaltando que hay casos en donde van hasta desde 4-15 (3\%) veces en temporada de tolvaneras. En contra parte, el $25 \%$ de los cuestionados no han tenido la necesidad de ir al médico. El costo promedio cuatrimestral que esto tiene es $29,8 €$ (ver tabla 3 ) porque la mayoría de las personas no cuentan con servicios médicos públicos que los apoyen y se ven en la necesidad de acudir a médicos privados, resaltando que existen casos que gastan hasta $209 €$ bajo este rubro.

FIGURA 4 - Porcentaje de asistencia médica personas/cuatrimestre

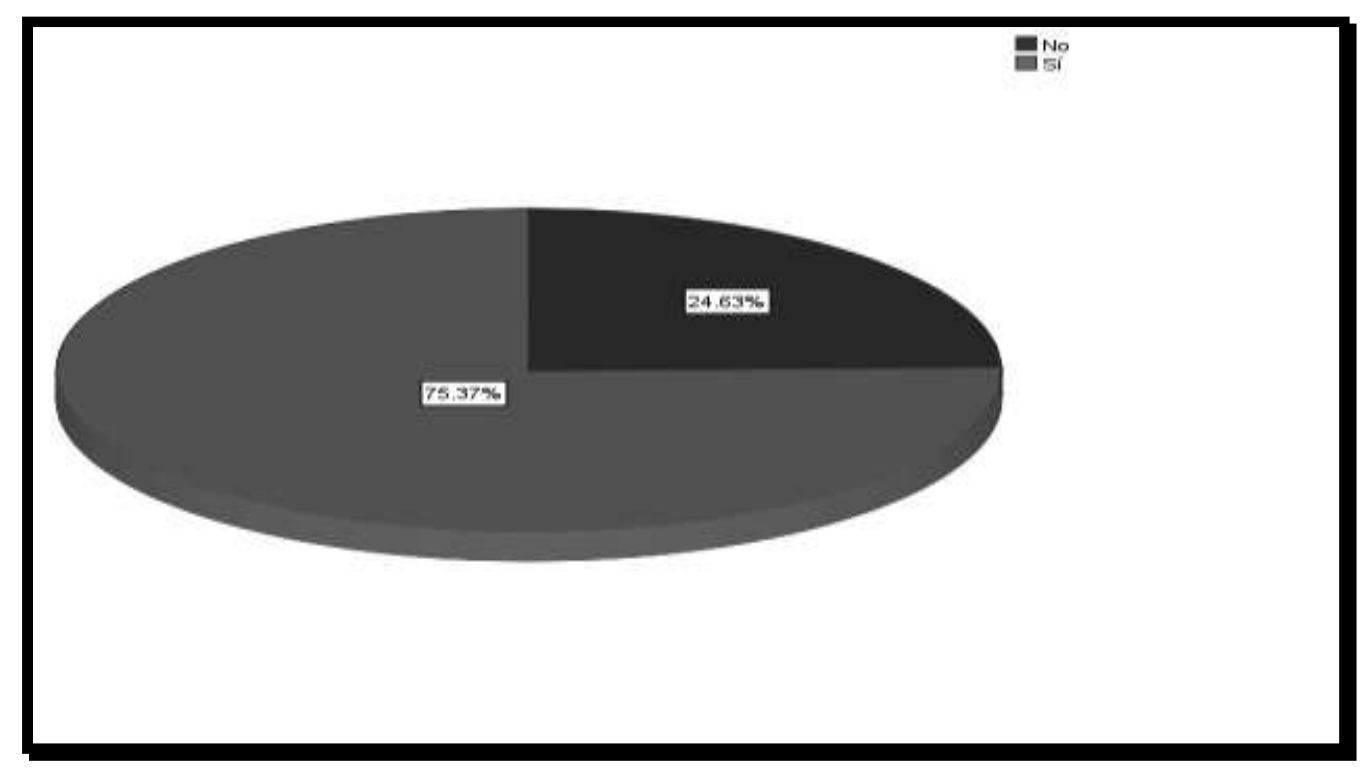

FUENTE: Elaboración propia con base al trabajo de campo.

Aunado a ello, en la tabla 3 se menciona que el costo promedio por cuatrimestre de las medicinas para contrarrestar esas enfermedades es $33 €$, sobresaliendo que hay registros de hasta $420 €$. Afortunadamente, la mayoría de los habitantes entrevistados (98\%) no han tenido necesidad de estar hospitalizados por estas causas, sin embargo, hay 5 registros $(2 \%)$ de personas que sí acudieron por ello, presentado un rango de costos que va $84-210 €$. Las personas mencionaban que cuando estaban hospitalizadas era al menos por dos noches en donde podían cobrarles hasta $42 €$ por cada una en un sitio privado. 
Campos Neutrais - Revista Latino-Americana de Relações Internacionais Vol. 1, No 2, p. 8-25, Maio - Agosto de 2019

Por otro lado, la figura 5 exhibe que $92 \%$ de la población interrogada se ve en la necesidad de usar tapabocas por el arribo de las tolvaneras, generando un costo promedio cuatrimestral de $6,3 €$ en donde la inversión puede ser desde 1,3€ como mínimo hasta llegar a $13,4 €$ como la cantidad más elevada. También es necesario señalar que $41 \%$ de los entrevistados no gastan en esto, porque la Secretaría de Salud Pública de Michoacán regala el producto o algunos de ellos cuentan con el apoyo del Instituto Mexicano del Seguro Social (IMSS) o Instituto de Seguridad y Servicios Sociales de los Trabajadores del Estado (ISSSTE).

En este orden de ideas, la figura 6 ilustra que $43 \%$ de los entrevistados han dejado de trabajar por las tolvaneras o las enfermedades, mientras $57 \%$ no pierden ningún día. Los habitantes de las localidades mencionaban que esto sucede así porque las personas tienen la necesidad de trabajar, aunque las condiciones no sean favorables en ese tiempo (enero-abril), y en ocasiones las actividades productivas las realizan en otros sitios lejos de la problemática. Se pierden en promedio por cuatrimestre 20 días de trabajo. Sin embargo, hubo comentarios en donde se indicaba que en ocasiones extremas se pierden hasta 72 días, esto evidencia que las tolvaneras impactan negativamente en la productividad laboral de las personas que habitan en la zona de estudio.

TABLA 3 - Costo total defensivo $€ /$ cuatrimestre/hogar

\begin{tabular}{|c|c|c|c|c|c|}
\hline Costos & Media & Mediana & Desviación Típica & Mínimo & Máximo \\
\hline Costo en consulta médica & $29,8 €$ & $16,7 €$ & $34,3 €$ & $2,5 €$ & $209 €$ \\
\hline Costo en medicina & $33 €$ & $16,7 €$ & $49,2 €$ & $2,9 €$ & $42 €$ \\
\hline Costo en tapabocas & $6,3 €$ & $6,3 €$ & $2,9 €$ & $1,3 €$ & $13,4 €$ \\
\hline $\begin{array}{c}\text { Costo total defensivo } \\
\text { por hogar }\end{array}$ & \multicolumn{5}{|c}{$69,1 €$} \\
\hline
\end{tabular}

FUENTE: Elaboración propia con base al trabajo de campo.

Los habitantes de las localidades recuerdan que las tolvaneras es un problema que se presenta desde el año 1960 durante el periodo de estiaje en el vaso Oeste del lacustre, pero se ha vivido con mayor intensidad a partir del año 2000 (42\%). Las personas recordaban que a raíz de la construcción de tres carreteras que dividen al lago en diferentes secciones la problemática se tornó más evidente y complicada. Desde entonces, el impacto ambiental es evidente. 
Campos Neutrais - Revista Latino-Americana de Relações Internacionais Vol. 1, No 2, p. 8-25, Maio - Agosto de 2019

FIGURA 5 - Porcentaje de personas/cuatrimestre que utilizan tapabocas

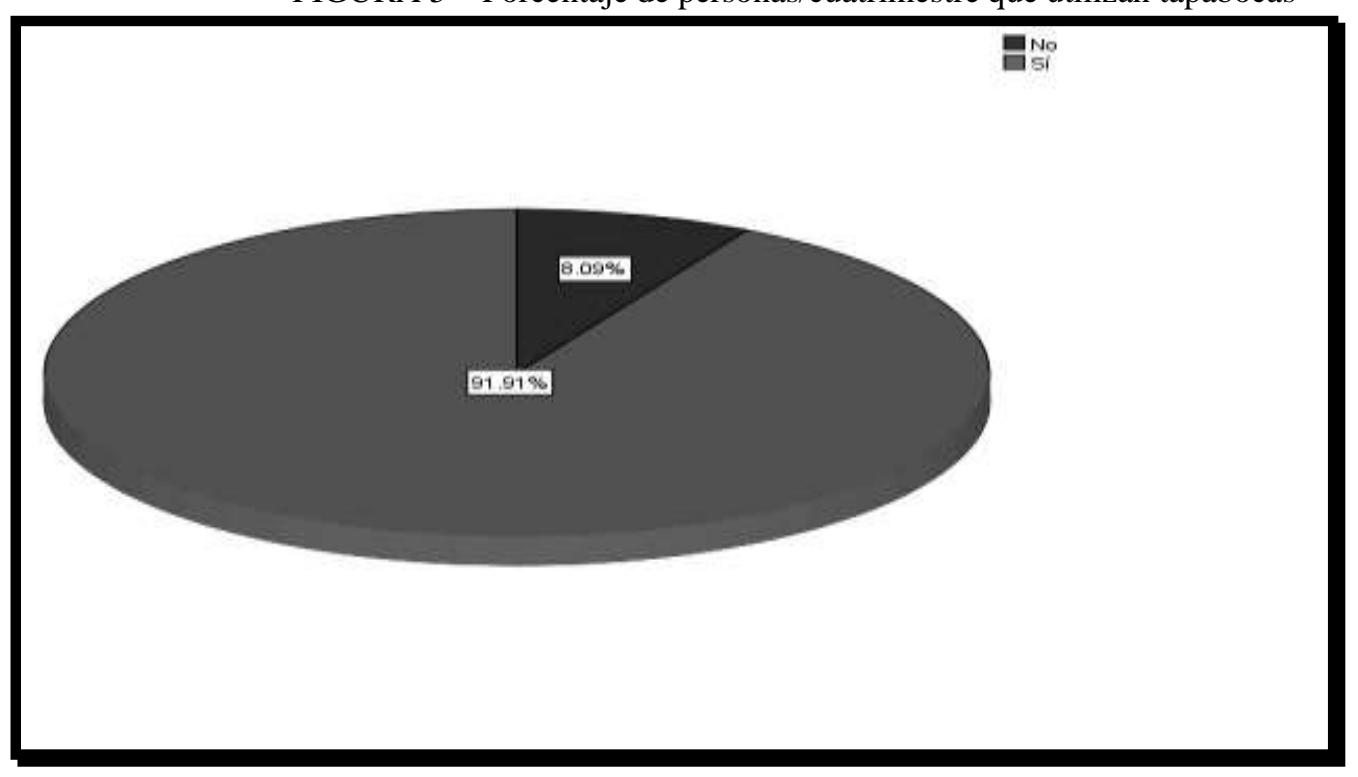

FUENTE: Elaboración propia con base al trabajo de campo.

FIGURA 6 - Porcentaje de personas entrevistadas que pierden días laborales por las tolvaneras

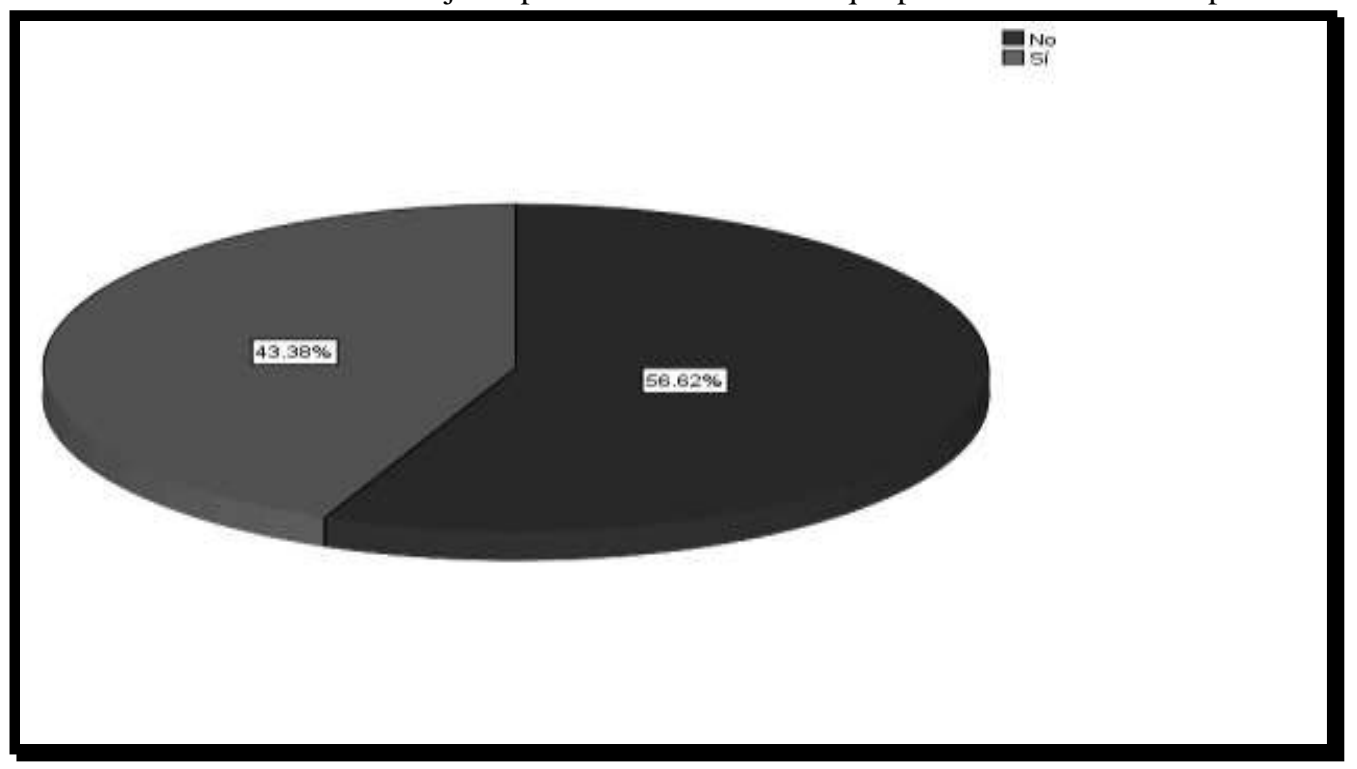

FUENTE: Elaboración propia con base al trabajo de campo.

Disposición a aceptar una compensación por la pérdida de bienestar en salud de los entrevistados en la Región Oeste del Lago de Cuitzeo

Ahora bien, en cuanto a la valoración económica por la pérdida del bienestar en salud que están sufriendo los habitantes de la región, se identifica que el 92\% de los interrogados tienen una DAC mediante un vale de gastos médicos a cambio del daño que les ocasiona el deterioro ambiental del lago por el arribo de las tolvaneras.

La cantidad mínima que aceptarían está en un rango de 20,9€ (21\%) a 84€ (40\%). Los entrevistados consideran que los principales motivos para aceptar tal cantidad de dinero son 
Campos Neutrais - Revista Latino-Americana de Relações Internacionais Vol. 1, No 2, p. 8-25, Maio - Agosto de 2019

porque: consideran apropiado que la compensación se haga mediante el vale (63\%), evitaría un gasto extra en salud (68\%) y es tema prioritario para la zona de estudio (61\%). En contra parte, el $8 \%$ de las personas no aceptaría la compensación porque no soluciona el problema, no consideran apropiado que el pago se realice como se menciona y la propuesta tiene falta de credibilidad.

\section{Disposición a pagar de los entrevistados por mejoras ambientales en la Región Oeste del}

\section{Lago de Cuitzeo}

En este apartado se analizaron algunas acciones para tratar de fomentar el saneamiento del lago y reducir el problema de las tolvaneras, en donde los entrevistados consideraron muy urgente con mayor frecuencia implantar vegetación en zonas fundamentales de la ribera del lago (97\%), reforestar en zonas fundamentales de la cuenca (95\%), ampliar la infraestructura de plantas de tratamiento para agua residual (92\%), implementar sistemas eficientes para la captación de agua (85\%) y en segundo plano, disminuir la utilización de agroquímicos en la cuenca (82\%), elaborar el compostaje de los desechos urbanos y rurales (67\%) y crear rellenos sanitarios intermunicipales apegados a las normas ambientales (62\%).

En esa orientación, los entrevistados (60\%) tienen una DAP mensual mediante el recibo de agua para realizar alguna de las acciones mencionadas. La cantidad máxima que pagarían se encuentra en un rango de $2,1 €$ a $4,2 €$. Los principales motivos son: tener la disponibilidad del lago para futuras generaciones (51\%), para mejorar las condiciones de salud (51\%), están a favor de la conservación y restauración del lago (50\%), para tener la disponibilidad de disfrutar el panorama del lago si se restaura (45\%) y porque viven cerca de la zona dañada (44\%).

En segundo término, para que las actividades económicas no se resientan (38\%) y porque su nivel de ingreso les permitiría pagar esa cantidad (33\%). Por otro lado, el $40 \%$ de la población interrogada no estaría dispuesta contribuir y los principales motivos son: el gobierno es quien debe aportar los fondos para mejorar el lago y disminuir las tolvaneras (37\%), porque su nivel de ingreso no permitiría pagar (35\%), y existe la incertidumbre de que el dinero se destine para otros fines $(20 \%)$.

\section{Perfil de los entrevistados en la Región Oeste del Lago de Cuitzeo}

Las características socioeconómicas de los entrevistados son las siguientes: tienen una edad promedio de 49 años, se cuestionó a 215 hombres y 57 mujeres. Este comportamiento se presentó así porque al momento de recolectar la información se buscaba platicar con el 
Campos Neutrais - Revista Latino-Americana de Relações Internacionais Vol. 1, No 2, p. 8-25, Maio - Agosto de 2019

encargado de la familia, quien normalmente en las localidades es un hombre. Sin embargo, sobre la marcha en la aplicación del cuestionario se detectó que los varones estaban en casa poco tiempo por estar laborando. Por ello, se eligió también entrevistar a las mujeres, asumiendo que ellas también conocen la información socioeconómica que se les preguntaba.

La cantidad de habitantes promedio es de 4. El grado de estudios oscila entre la primaria (33\%) y secundaria (28\%). Los años en promedio que tiene viviendo en la localidad son 46 y en su vivienda 33. En general, la mayoría de la población entrevistada mencionaba que la gente que no se encontraba en sus casas era porque habían tomado la decisión de migrar a los Estados Unidos, porque no encontraban condiciones laborales aceptables y por la problemática de las tolvaneras.

Los entrevistados se dedican a la construcción (36\%), al comercio (28\%), y a otras actividades (12\%), en donde destacan empleada doméstica, artesano en carrizo, músicos, etc. En la familia trabajan entre una $(20 \%)$ o dos $(6 \%)$ personas a parte de la persona responsable de la vivienda. Trabajan alrededor de $6(29 \%)$ y 7 (47\%) días a la semana, y en un rango de 7 $(15 \%)$ u $8(27 \%)$ horas al día. El ingreso mensual personal es menos de 83,5€ y el familiar oscila de 83,5-166,7€. Todas estas características indican que la población que habita en las localidades ribereñas al lago, se enfrenta a un escenario de condiciones socioeconómicas poco favorables teniendo en cuenta la cantidad monetaria que deben invertir en salud para sobreponerse a las externalidades ambientales que les ocasiona la contaminación del lago de Cuitzeo.

\section{Conclusiones}

El costo defensivo total al que incurre una familia por hogar en un cuatrimestre por el arribo de las tolvaneras es $69,1 €$. Distribuidos en costo promedio en consultas médicas $(29,8 €)$, costo promedio en medicinas $(33 €)$ y costo promedio en tapabocas $(6,3 €)$. Existe una disminución en la productividad laboral por el arribo del polvo, ya que se pierden 20 días en promedio durante un cuatrimestre por el deterioro ambiental que presenta el lago de Cuitzeo.

Existe una DAC por los habitantes entrevistados mediante un vale de gastos médicos para solventar los costos defensivos que están sufriendo por la pérdida de bienestar en salud atribuido a la externalidad negativa del arribo de las tolvaneras. La cantidad mínima que aceptarían está en un rango de 20,9-84€.

Los principales motivos para aceptar la ayuda económica son porque evitaría un gasto extra en salud y se necesita el apoyo en la zona. Según los consultados las acciones que tienen prioridad para implementarse en búsqueda de fomentar el saneamiento del lago y reducir las 
Campos Neutrais - Revista Latino-Americana de Relações Internacionais Vol. 1, No 2, p. 8-25, Maio - Agosto de 2019

tolvaneras son: ampliar la infraestructura de plantas de tratamiento para agua residual y reforestar e implantar vegetación en zonas estratégicas de la cuenca, haciendo más énfasis en el lago. En esos términos, la vegetación y los árboles deben obedecer a las características específicas que posee el suelo degradado.

Existe una DAP por los entrevistados para realizar alguna de las acciones mencionadas para mejorar las condiciones ambientales del lacustre, la cantidad máxima está en un rango de 2,1-4,2€. Los principales motivos para realizar la contribución mediante el recibo de agua son: para mejorar la salud, porque están a favor de la conservación y restauración del lago y por disfrutar del panorama que brindaría el lago si se restaura.

Las preferencias sociales y la valoración monetaria asignada para los mercados hipotéticos de la DAC y DAP en esta investigación, se desarrolla de manera instrumental. Esto indica que las propuestas sólo son elementos de gestión cuyo valor refleja los costos de la poca intervención de la población para avanzar en resolver una problemática ambiental compleja como sucede en el lago de Cuitzeo.

\section{Bibliografía}

AGUILERA, Federico y ALCÁNTARA, Vicent. De la economía ambiental a la ecológica. Barcelona: Fuhem e Icara, 1994.

AZQUETA, Diego. Introducción a la Economía Ambiental. Madrid: McGraw-Hill, 2002.

BRAVO-ESPINOSA, Miguel, BARRERA-CAMACHO, Gerardo, MENDOZA, Manuel, SÁENZ, Trinidad, BAHENA-JUÁREZ, Fernando, y SÁNCHEZ-MARTÍNEZ, Rubén. Contribuciones para el desarrollo sostenible de la cuenca del Lago de Cuitzeo, Michoacán. México: Centro de Investigaciones sobre Geografía Ambiental, 2012

COASE, Ronald. The problemof social cost. The Journal of Law and Economics, vol. III, pp. 1-44. SystemsResearch, 1960.

CHACÓN, Arturo, ROSAS, Catalina, y ALVARADO, Javier. El lago de Cuitzeo. En DE LA LANZA, Guadalupe (Org.). Las aguas interiores de México, 2007, p. 304-360.

FERNÁNDEZ, Pilar, SOMMER, Irene, PONCE DE LÉON, Claudia, DÍAZ, Carlos, BECERRA, Veronica y CRAM, Silke. El Lago de Cuitzeo: Elementos contaminantes al interior del lago. En CRAM, Silke, GALICIA, Leopoldo y ALCÁNTARA, Israde (Org.). Atlas de la cuenca del lago de Cuitzeo: análisis de su geografía y su entorno socioambiental. Instituto de Geografía-UNAM/Universidad Michoacana de San Nicolás de Hidalgo, México, 2010, p. 246-249.

FIELD, Barry. Economía y medio ambiente. Santafé de Bogota: McGRAW-HILL, 1996.

INEGI. Prontuario de información geográfica municipal de los Estados Unidos 
Campos Neutrais - Revista Latino-Americana de Relações Internacionais

Vol. 1, No 2, p. 8-25, Maio - Agosto de 2019

Mexicanos.México. Obtenido de

http://www3.inegi.org.mx/contenidos/app/mexicocifras/datos_geograficos/16/16020/2009.pdf , acceso el 05 de Marzo, 2017.

INEGI. Censo de Población y Vivienda. Obtenido de http://www.beta.inegi.org.mx/proyectos/ccpv, acceso el 02 de Ma

Labandeira, Xavier, León, Carmelo, y Vazquéz, María. Economía Ambiental. Madrid: Pearson Educación S.A., 2007.

MALONEY, Michael, WARD, Michael, BRAUCHT, Nicholas. A revised scale for the measurement of ecological attitudes and knowledge. American psychologist, vol. 30, no. 7, p. $787,1975$.

MARTÍNEZ-PAZ, Jóse Miguel, PERNI-LLORENTE, Ángel, y ALMANSA-SÁEZ, Carmen. Valoración económica del parque regional Sierra Espuña. Espacios Naturales Protegidos en la Región Murcia, p. 1-20, 2010.

MARTÍNEZ-PAZ, José Miguel, ALMANSA-SÁEZ, Carmen, y PERNI-LLORENTE, Ángel. Energía eléctrica procedente de fuentes renovables: Percepción social y disposición al pago.

Estudios de EconomíaAplicada, vol. 29, no. 2, p. 6-21, 2011.

PEARCE, David. The limits of cost-benefit analysis as a guide to environmental policy.

Kyklos, vol. 29, no. 1, p. 97-112, 1976.

PIGOU, Arthur. La economía del bienestar. Ed. Aguilar, Madrid, 1962.

RIERA, Pere. Manual de Valoración Contingente. Ministerio de Economía y Hacienda, Instituto de Estudios Fiscales, 1994.

Secretaria de Salud en Michoacán. Jurisdicción Sanitaria Nº1 Morelia. Carpeta Básica. Coordinación y evaluación y Estadística, 2015.

SUMA. Saneamiento integral para impulsar el desarrollo agropecuario y social en la Cuenca del Lago de Cuitzeo. Morelia, Miachoacán, México, 2008.

TRUEBA, Rafael y ORTIZ, Carlos. Métodos de valoración económica vinculados al análisis costo-beneficio extendido: el caso de la Región Oeste del Lago de Cuitzeo, Michoacán, México. Desarrollo local sostenible, vol.12, no.34, p. 1-21, 2019. 